\title{
Review on Effects of Electrode in Electrical Discharge Machining Process
}

\author{
Nayan J. Patel \\ Department of Mechanical Engineering, Parul Institute of Engineering and Technology (Diploma Studies), \\ Parul University, Waghodia, India
}

\begin{abstract}
Electrical Discharge Machining is one of the non-conventional machining processes used for electrically conductive material. It is widely used for manufacturing complicated parts which are tough to be produced by conventional manufacturing processes. It is based on thermoelectric energy between workpiece and electrode. Metal is removed by melting and vaporizing because of spark occurs in the gap between electrode and workpiece. Workpiece and electrode must have electrically conductive to generate a spark. The performance of the EDM process is largely depends on the electrode. Electrode is considered as tool in EDM process. Selection of the electrode material plays vital role in the EDM process. Different electrode materials have different properties. Hence, the performance of the EDM process changes with different materials. Researchers have used different materials as electrode to investigate the effects of materials and to improve the performance of EDM process. This paper reviews the research work carried out in the field of materials and manufacturing methods for electrodes in EDM process.
\end{abstract}

Keywords: [EDM, Electrodes, Materials, Manufacturing Process]

\section{INTRODUCTION}

Electrical discharge machining is well established machining alternative for manufacturing geometrically complex parts from electrically conductive materials which are hard to be machined by conventional process. Any hard material which is electrically conductive can be easily machined by EDM process. Because of this advantage, it is widely used for manufacturing dies, automotive parts, surgical and aerospace components. There is no direct contact between tool electrode and workpiece. It eliminates chatter, vibrations and mechanical problems during machining.

In sinker EDM process, electrode and workpiece are submerged into dielectric fluid such as kerosene. Power supply is connected to electrode and workpiece. As electrode approaches the workpiece, dielectric breakdown occurs which forms plasma channel and a small spark jumps. This spark hits one at time. Sparks occurs in large number at asperities. When base metal is eroded, spark gap increases. Electrodes lowered automatically by servo control system in EDM.

Electrode is considered as tool in EDM process. The main role of electrode is to pass electrical current to allow erosion of workpiece. The performance of EDM process largely depends on material and manufacturing process of electrode.

\section{Materials used for EDM electrodes}

Tool material should not be worn faster when it is impinged by positive ions. The basic characteristics of electrode materials are high electrical and thermal conductivity, high melting point, high density, easy manufacturability and low cost.

Selection of material used for electrode plays very important role in EDM process because it affects the material removal rate, electrode wear rate and surface roughness (Haron et al., 2008). 
Electrode materials are generally classified into metal, non-metals and composites. Various materials used for EDM electrode are as follows:

Copper- Pure copper is a very important tool material when fine surface finish is required on the workpiece. The tool can be easily fabricated by casting or machining. Copper is generally recommended for holes and slots but not recommended for high accuracy and details.

Brass- Brass is often used as an electrode material generally for drilling small holes. It is easily available and can be quickly machined. Brass cannot be mainly used for machining hard materials. Brass is not recommended for high accuracy work.

Copper tungsten- Copper tungsten is a sintered electrode in which copper infiltrates the tungsten powder. It shows good wear rate and produce excellent surface finish. It is generally used for making small and medium sized electrodes of complex shape. Copper tungsten is not recommended for large areas. It has high strength so electrode does not show failure when machined into thin sections with fine details.

Copper infiltrated graphite- It shows better rigidity than graphite and combines the properties of both copper and graphite. It shows good performance under poor flushing condition and is good for machining tungsten carbide. The main disadvantage of the copper graphite is its higher corner wear rate and high cost.

Graphite- Graphite is best non-metal material used as electrode. It is most widely used electrode material in this era. It is comparatively cheap and easily available and shows best stability. It can be easily formed by machining, moulding, grinding etc. Graphite however has a property of arcing and may need anti arcing devices to be incorporated in set up.

\section{LITERATURE REVIEW}

Effect of EDM electrodes materials on performance of EDM process

Singh et al. (2004) worked to find out the most suitable electrode material for the electrical discharge machining of hardened En-31 tool steel because heat treated steel is hard to machining with traditional process. Different electrode materials used are copper, copper tungsten, brass and aluminium. With increasing the discharge current, $\mathrm{Cu}$ and Aluminium shows best MRR. With increase in discharge current MRR increases because spark discharged energy increases which cause the large impulsive force in spark gap. $\mathrm{Cu}$ and $\mathrm{Cu}-\mathrm{W}$ shows less wear. Aluminium and brass shows more wear with increase in discharge current. Aluminium and copper shows high MRR and also large craters. So it shows poor surface finish. Wong et al. (2001) investigated the effect of $\mathrm{TiC}$ in $\mathrm{Cu}-$ $\mathrm{W}$ electrode on electrical discharge machining of tool steel ASSAB 705 workpiece. If copper content increases, wear resistance decreases because low melting point of copper. If tungsten content is more then it increases porosity because of insolubility between copper and tungsten. TiC is used in it because it is high refractory material with high melting point and high thermal shock and abrasion resistance. $\mathrm{Ni}$ is also added because of its good solubility with copper and tungsten at liquid phase. Composition used in this work is Ni- 3.50 $\%, \mathrm{Cu}-25 \%, \mathrm{~W}+\mathrm{TiC}-75 \%$. There are six batches electrode which has different composition of $\mathrm{W}$ and TiC. Percentages of Tic in six batches of are 5,10,15,20,30,60 and percentages of $\mathrm{W}$ are $95,90,85,80,70,40$ which called A1, A2, A3, A4 A5 and A6 respectively. Densification of $\mathrm{Cu}-\mathrm{W}$ electrode improved by addition $\mathrm{Ni}$ which was verified from XRD chart. Electrical resistivity of electrode of $15 \% \mathrm{TiC}$ is less because at that higher relative density and lower porosity. So it gives higher MRR and lower EWR. At $15 \%$ TiC, relative density is high, so porosity is lower decreases the electrical resistance and create strong bonding between particles. So, it shows high MRR and low EWR. At higher TiC percentage, increasing porosity made weak bonding between particles and hence particles drop out during machining which 
makes the spark scattered and reduces the strength of spark, which also increases surface roughness.

Haron et al. (2008) investigated the effect of copper and graphite electrode with different diameters for machining of XW 42 tool steel and to select optimum material. High tool wear is main problem in EDM which result in inaccurate dimension. So research was done to select the optimum material combination and electrode material. Different diameters used for electrode in $\mathrm{mm}$ are 10, 15, 20 and different current intensity used in ampere is $3 \mathrm{~A}$ and 6A. Dielectric used is kerosene. The mass lost from electrode and work material is weighed by digital weighing scale. Copper and graphite used have melting point $1083^{\circ} \mathrm{C}$ and $455^{\circ} \mathrm{C}$. EWR of $\mathrm{Cu}$ electrode is lower than graphite electrode. $\mathrm{Cu}$ has higher melting point and wear resistance, so less eroding of material which reduces EWR. Electrode wear rate increases with increasing current intensity. The ratio of mass loss percentage between higher current setting and lower current setting is up to 10 times for graphite electrode and 15 times for copper electrode. For copper electrode EWR decreases with increasing size of electrode. Material removal rate is more at high current for both electrodes. MRR decreases with increasing size of electrode for copper, while for graphite $15 \mathrm{~mm}$ diameter electrode shows highest MRR. They found that found that the bigger electrode diameter will lose more heat when machining, because this heat will be transferred to electric fluid. The temperature of electric fluid will increase close to the melting point of the material. Copper is suitable as electrode material for machining of XW42 tool steel because it shows higher MRR and lower EWR than graphite electrode. Muttamara et al. (2009) investigated the effect of copper, graphite (Poco EDM - 3) and copper infiltrated graphite (Poco EDM-C3) electrodes on generation of conductive layer on alumina during Electrical Discharge Machining. In electrical discharge machining of alumina, electrical conductive layer does not form sufficiently to keep stable machining and continuous discharge, so carbon backed layer is used as assisting electrode. EDMC3 with positive polarity shows highest material removal rate and lowest electrode wear ratio, while solid copper electrode shows lower material removal rate and highest electrode wear ratio. These results show material removal rate is more with positive polarity which is same as for EDM on conductive materials. Thickness of conductive layer plays an important role in EDM process. In both case of EDM with positive and negative polarity, EDM-C3 gives thickest conductive layer followed by EDM-3. Electrical resistivity of conductive layer generated by EDM-C3 gives lowest value which causes more material removal rate. Values of the electrical resistivity of conductive layer generated by copper and EDM-3 electrode are quite similar but EDM-C3 shows higher material removal rate than copper. It shows that thickness of conductive layer seems to affect material removal rate.

\section{Effect of manufacturing methods used for EDM electrodes on EDM process}

Zhao et al. (2003) used electrode made by selective laser sintering process for electrical discharge machining of 45\# steel. Major cost and time spent in EDM process is manufacturing electrode. But with the help of the rapid prototyping manufacturing technology, it is possible to reduce lead time and development cost. Multi component powder consists of steel, polyester and phosphate is used as material for selective laser sintering. Here, steel powder is base material, polyester is binder and phosphate reacts with steel to form inorganic compound material which is rigid. Post treatment is done to improve strength and density of prototype. There are three types of samples used with different proportions of materials. The input parameters like pulse interval 195, 380, 500, $750 \mu \mathrm{s}$, pulse duration 66 and $250 \mu$ s and current 4, 6 and 10 amperes are selected. Electrode wear rate for sample no. 1 and no. 2 is increased with 
increase in pulse interval, while for sample no. 3, it is stable. The density of the electrode is determined by base material which is steel in this case. The greater is steel, more uniform is pore distribution and less is the quantity of copper infiltrated. Sample no. 3 has more density than sample no. 1 and no. 2. So it shows less tool wear. It shows the potential of machining using an electrode made by selective laser sintering. Beri et al. (2014) improved micro hardness of machined surface by using powder metallurgy processed electrode. Conventional methods of fabricating electrodes require long processing time and results in materials wastage when complex geometry produced. In this research work, Inconel 718 alloy steel was selected as workpiece, electrodes used were cylindrical copper electrode and powder metallurgy processed CuW2575 and CuW2080. CuW2080 showed maximum micro hardness. Micrograph taken of the cross section of machined surface which shows formation of deposited layer, recast layer and heat affected zone. Layer of order of $28.71 \mu \mathrm{m}$ deposited on work surface and causes a modification of machined surface called surface alloying. XRD analysis of machined work surface was performed to find out nature of compounds formed after electrical discharge machining with CuW2080 electrode. It shows that compounds like Fe6W6C, Cr2F14C and $\mathrm{NMo} 4 \mathrm{C}$ are formed. It is also observed that almost all peaks show formation of Fe6W6C phase which confirms that tungsten gets detached from electrode and forms iron tungsten carbide which is hard. Formation of Fe6W6C phase resulted in surface coating of machined surface which increases micro hardness of surface. Tsai et al. (2003) proposed new method of blending the copper powder contained resin with chromium powder to form tool electrode at low temperature and low pressure in hot mounting machine. Normally, electrodes are fabricated at high temperature and pressure. Method proposed in this paper is fast, simple and less costly. Aim of paper is to improve the corrosion resistance. Workpiece used in this research work was 1045 medium carbon tool steel. Sintering pressure used for electrode were $10 \mathrm{MPa}, 20$ $\mathrm{MPa}$ and $30 \mathrm{MPa}$. Different compositions used for electrode fabrication are $\mathrm{Cu}-100 \%$ $\mathrm{Cr}-0 \%, \mathrm{Cu}-80 \% \mathrm{Cr}-20 \%$ and $\mathrm{Cu}-57 \%$ Cr- $43 \%$. Cu-100 \% Cr-0\% electrode shows highest material removal rate and $\mathrm{Cu}-57 \%$ Cr- $43 \%$ shows lowest material removal rate. This is because weaker bonding strength between $\mathrm{Cu}$ and $\mathrm{Cr}$ particles. It also shows that positive polarity machining always obtained higher material removal rate than that obtained by negative polarity machining. Material removal rate increases with sintering pressure. At low sinter pressure, density of the composite electrodes decreases, so it showed low material removal rate. By comparison between conventional $\mathrm{Cu}$ electrode and $\mathrm{Cu}$ $\mathrm{Cr}$ composite electrode, it was found that higher material removal rate is obtained with composite electrode than conventional $\mathrm{Cu}$ electrode. Khanra et al. (2007) developed metal matrix composite (ZrB2$\mathrm{Cu})$ to get optimum combination of wear resistance, electrical and thermal conductivity. High tool wear is major drawback of electrical discharge machining. So aim of paper is to develop a tool material which has high electrical and thermal conductivity, high wear resistance and easy fabric ability and availability. Here, ZrB2$\mathrm{Cu}$ composite electrode prepared through liquid phase sintering. Workpiece material was mild steel. $\mathrm{ZrB} 2-\mathrm{Cu}$ composite electrode with $40 \% \mathrm{Cu}$ shows higher material removal rate and comparatively lower tool removal rate. Relative density increases with $\mathrm{Cu}$ addition due to decrease of porosity. $40 \%$ wt $\mathrm{Cu}-\mathrm{ZrB} 2$ considered as " $A$ " tool and its performance is compared to $\mathrm{Cu}$ tool for pulse on time and different current. "A" tool shows higher material removal rate and lower tool removal rate than $\mathrm{Cu}$ tool which indicate higher wear resistance of " $A$ " tool due to presence of ZrB2 particles. X-ray diffraction shows formation of $\mathrm{ZrC}$ as new phase after EDM. 
At high temperature during EDM, results in partial dissociation of $\mathrm{ZrB} 2$ into $\mathrm{Zr}$ and $\mathrm{B}$ and then $\mathrm{Zr}$ react with $\mathrm{C}$ which formed due to breakdown of dielectric, forms $\mathrm{ZrC}$ phase which is hard phase and increase wear resistance composite which leading to lower tool removal rate. So, $40 \%$ wt $\mathrm{Cu}-\mathrm{ZrB} 2$ made by liquid phase sintering is selected as best electrode because its performance is better than $\mathrm{Cu}$ electrode.

\section{CONCLUSION}

It has been discussed research work done by different authors in the field of electrical discharge machining. Many researchers worked on the improving performance of EDM by using different electrode materials such as copper, copper tungsten, brass, aluminium etc. Some researchers also used composite electrodes like $\mathrm{Cu}-\mathrm{Cr}, \mathrm{ZrB} 2-\mathrm{Cu}$ etc. Different manufacturing methods used for electrodes have also been discussed. Cost of manufacturing electrode is about $50 \%$ of total cost of EDM process. Powder metallurgy processed electrode shows better performance than electrode manufactured by conventional methods. Cost of the electrode manufacturing can be reduced by powder metallurgy because with single die and punch assembly, number of electrodes cam be produced which also reduces overall cost of EDM process. EDM is useful for machining advanced materials like hardened tool steel, Inconel 718 alloys which are difficult to machine with conventional machining processes. There is possibility to investigate effect of electrodes manufactured by powder metallurgy process using different electrode materials while EDM of some advanced materials like Inconel alloys, tool steels. Electrode affects the performance of EDM process. It is very important part of EDM process.

Acknowledgement: None

Conflict of Interest: None

\section{Source of Funding: None}

\section{REFERENCES}

1. N. Beri, S. Maheshwari, C. Sharma, A. Kumar, 2014, Surface quality modification using powder metallurgy processed $\mathrm{CuW}$ electrode during electrical discharge machining of Inconel 718, Procedia Materials Science 5, pp.2629-2634

2. Bhola Jha, K.Ram, Mohan Rao, 2011, An overview of technology and research in electrode design and manufacturing in sinking electrical discharge machining, Journal of Engineering Science and Technology Review 4 (2), pp. 118-130

3. A. Muttamara, Y. Fukuzawa, N. Mohri, T. Tani, 2009, Effect of electrode material on electrical discharge machining of alumina, Journal of Materials Processing Technology 209, pp.2545-2552

4. C. haron, J. Ghami, Y. Burhanuddin, Y.K. Seong, C.Y. Swee, 2008, Copper and graphite electrodes performance in electrical discharge machining of XW42 tool steel, journal of materials processing technology 201, pp. 570-573

5. A. Khanra, B. Sarkar, B. Bhattacharya, L. Pathak, M, Godkhindi, 2007, Performance of $\mathrm{ZrB} 2-\mathrm{Cu}$ composite as EDM electrode, Journal of materials processing technology 183 , pp. $122-126$

6. S. Singh, S. Maheshwari, P. Pandey, 2004, Some investigations into the electric discharge machining of hardened tool steel using different electrode materials, Journal of materials processing technology 149 , pp. 272-277

7. H. Tsai, B. Yan, F. Huang, 2003, EDM performance of $\mathrm{Cr} / \mathrm{Cu}$ based composite electrodes, International journal of machine tools \& manufacture 43, pp. 245-252

8. Li Li, Y. Wong, J. Fuh, Li Lu, 2001, Effect of $\mathrm{TiC}$ in copper-tungsten electrodes on EDM performance, Journal of materials processing technology 113 , pp. 563-567

How to cite this article: Patel NJ. Review on effects of electrode in electrical discharge machining process. International Journal of Research and Review. 2021; 8(5): 91-95. DOI: https://doi.org/10.52403/ijrr.20210513 